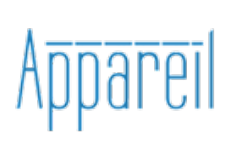

Appareil

$11 \mid 2013$

L'espace et l'architecture: état des lieux

\title{
La notion d'appareil empruntée à W. Benjamin, appliquée à la création musicale en studio électroacoustique, et à sa diffusion
}

\section{Anne Sèdes}

\section{(2) OpenEdition}

Journals

Édition électronique

URL : http://journals.openedition.org/appareil/1772

DOI : 10.4000/appareil. 1772

ISSN : 2101-0714

Éditeur

MSH Paris Nord

Référence électronique

Anne Sèdes, «La notion d'appareil empruntée à W. Benjamin, appliquée à la création musicale en studio électroacoustique, et à sa diffusion », Appareil [En ligne], 11 | 2013, mis en ligne le 26 septembre 2013, consulté le 30 juillet 2020. URL : http://journals.openedition.org/appareil/1772 ; DOI : https:// doi.org/10.4000/appareil.1772

Ce document a été généré automatiquement le 30 juillet 2020.

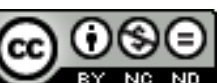

Appareil est mis à disposition selon les termes de la Licence Creative Commons Attribution - Pas d'Utilisation Commerciale - Pas de Modification 4.0 International. 


\title{
La notion d'appareil empruntée à W. Benjamin, appliquée à la création musicale en studio électroacoustique, et à sa diffusion
}

\author{
Anne Sèdes
}

1 En quoi la notion d'appareil empruntée à Benjamin peut être utile aux musiciens et musicologues, pour examiner des objets d'études, des faits musicaux, des manières de faire, des poïétiques, qu'elles viennent du répertoire contemporain dit «savant » ou des musiques dites "populaires». Comment une réflexion sur la notion d'appareils peut nous aider à renouveler nos approches?

\section{De Walter Benjamin à Pierre Schaeffer}

2 Ce que pointe Benjamin, à propos des appareils, concerne le cinéma, la photographie, et dans sa généralité, l'œuvre d'art à l'époque de sa reproductibilité technique.

Benjamin s'intéresse à la caméra, aux microphones, aux éclairages présents sur le plateau de tournage cinématographique, et qui constituent l'appareillage entre l'acteur et les experts, entre l'acteur et les masses. Il évoque le cinéma en tant que système, soulignant le point de vue de l'acteur, le point de vue de l'équipe de tournage et des experts, le point de vue des masses, les contraintes de production et de diffusion, la publicité, la starisation, dans le contexte historique et politique d'une époque donnée.

4 La réflexion sur l'appareil, et comment les mutations technologiques, en interaction avec l'époque, transforment l'art, nos manières de faire et de percevoir, c'est là tout l'éclairage du texte de Benjamin.

5 André L. Villa de Almeida souligne dans sa thèse ${ }^{1}$ la contemporanéité de trois textes :

6 - L'ouvre d'art à l'époque de sa reproductibilité technique de Walter Benjamin (rédigé entre 1935-1936) 
- Du fétichisme en musique et de la régression de l'audition de Theodor W. Ardorno ${ }^{3}$;

- Vingt leçons et travaux pratiques destinés aux musiciens mélangeurs de Pierre Schaeffer ${ }^{4}$.

Si Adorno est du côté de la critique, pointant la régression de l'audition face au développement des industries musicales, Benjamin est du côté de l'ouverture d'un Schaeffer s'appropriant l'usage des appareils de mixage du son pour la radio, à des fins musicales, invitant à former des «musiciens mélangeurs " plutôt que des techniciens $\mathrm{du}$ son, preneurs de son ou mixeurs. Cette démarche mènera, à l'après-guerre, Schaeffer à l'invention de la « musique concrète », à partir de l'enregistrement et de la manipulation de sons complexes et préexistants à l'œuvre, des sons appartenant jusqu'alors à la catégorie du bruit ; une musique nouvelle s'impose, avec de nouvelles manières de faire et d'écouter, d'après les moyens et l'appareillage de la radiodiffusion. La musique concrète s'est perpétuée jusqu'à nos jours à travers les courants des musiques électroacoustiques, acousmatiques et mixtes dans un contexte de création expérimentale, construisant un nouveau style d'écoute et de production de contenus sonores désormais acceptés comme étant de la musique, jusqu'à influencer dans l'écriture la musique contemporaine instrumentale. Le compositeur allemand Helmut Lachenmann écrit ainsi une « musique concrète instrumentale ».

10 A. L. Villa de Almeida consacre un chapitre de sa thèse à questionner comment les mutations technologiques et l'appropriation que nous en faisons transforment nos styles d'écoute et nos manières de penser et de faire de la musique ${ }^{5}$ Villa commence sa revue par le moment du phonographe et de l'invention du disque pour la clore sur celui de la guitare électrique, dont certaines caractéristiques telles que la saturation et le feedback, d'abord comprises comme des désagréments, vont s'imposer jusqu'à nos jours comme des moyens d'expression musicale évidents.

\section{De la guitare électrique au studio de musique électroacoustique}

11 J'ai eu l'occasion de publier dans la revue Appareil, $\mathrm{n}^{\circ}$ 5, les actes de journées d'étude sur les «identités de la guitare électrique ${ }^{6}$. La notion d'appareil convenait exactement à cet instrument qui, au gré des appropriations par les musiciens, en faisant apparaître la multiplicité de ses identités esthétiques, a selon l'expression de Jean-Louis Déotte appareillé l'époque.

12 La guitare électrique n'est plus un instrument de musique au sens traditionnel du terme. C'est un instrument modulaire, un appareillage en réseau, fait d'une guitare à corps plein, équipée de microphones, connectée à un amplificateur qui va ajouter des possibilités de transformation et de traitement du son. Des pédales d'effet vont enfin compléter la chaîne de traitement électroacoustique. De nos jours, cette chaîne peut intégrer les moyens du traitement audionumérique.

13 C'est une banalité que de souligner l'importance de la guitare électrique, dans la construction $\mathrm{du}$ son des musiques pop, interagissant avec les moyens du studio d'enregistrement, tels que la réverbération et des techniques de ligne à retard, des premiers enregistrements du rockabilly jusqu'à nos jours.

14 Avec ses propriétés modulaires, en tant qu'appareil connectable à d'autres appareils, un appareillage, un réseau d'objets matériels, mais de nos jours aussi logiciels, la guitare électrique est également un instrument de studio. Elle nous renvoie aux 
pratiques des musiciens électroacousticiens, à leur manière de faire, à la façon dont ils manient ces appareils, les prennent en main, se les approprient pour produire des contenus musicaux.

15 Le studio d'enregistrement et de production est un ensemble d'appareils : des micros, des enregistreurs, des mixeurs, des filtres et des modules de délai, chambre d'écho, réverbérations... Un peu comme la guitare électrique (instrument, pédales, amplis), c'est un ensemble de modules, connectables entre eux. Un appareillage en réseau, de nos jours à la fois matériel et logiciel constituant l'environnement de travail des compositeurs.

On pourrait approcher les pratiques du studio par leurs pôles extrêmes: la musique expérimentale, qui se pratique plutôt de façon autonome, et la musique commerciale, dédiée aux industries de la culture. La musique expérimentale, sous la forme de la musique électroacoustique, est un objet d'étude traditionnel pour la musicologie. Pour aborder l'examen de ses manières de faire, certains ont avancé l'idée d'espaces composables, construits par le compositeur, en tant que son environnement conceptuel, très lié aux appareils, matériels, logiciels, conceptuels.

\section{Espaces composables}

17 La notion d'espace composable a été proposée par Horacio Vaggione, pointant les perspectives de la composition électroacoustique à l'heure du numérique ${ }^{7}$.

Vaggione examine l'espace interne du son musical, de ses morphologies, comment les articuler, les varier, et souligne que les moyens apportés par le numérique nous permettent d'opérer encore plus finement sur ces articulations, à des niveaux temporels multiples et très fins, comme avec autant de « manettes ».

Cette approche a été ensuite reprise et développée par Guilherme Carvalho ${ }^{8}$.

Un espace composable est défini par l'ensemble des opérations et des variables que permet cet espace. Les ensembles d'espaces nous permettent de composer des objets musicaux qui sont d'ailleurs eux même des espaces composables. Cette façon de "formaliser la forme" musicale qui se déploie chez Carvalho, dans un cadre géométrique et algébrique propre, procède là encore d'une pensée en réseau, des réseaux d'objets qui au fur et à mesure de la composition vont construire la forme musicale. Guilherme Carvalho démontre ainsi qu'un objet musical, une musique, est produit en fonction de l'espace de formalisation, de l'espace conceptuel qui a permis sa production, un espace défini par le compositeur.

21 L'espace composable du studio de musique expérimentale se définit par l'ensemble des variables et des opérations (de transformation sonore) que l'on effectue dans un environnement de travail donné. En studio, c'est l'interaction du compositeur avec son environnement de travail, avec les appareils, les systèmes, qu'ils soient de nos jours matériels ou logiciels, qui fait émerger les espaces composables, les manettes, les moyens d'opérer sur des objets et d'interagir avec les variables.

Ceci vaut en musique instrumentale également. Pour Helmut Lachenmann, composer, c'est réfléchir sur les moyens. C'est faire le choix de l'appareillage.

C'est définir son instrument, c'est-à-dire définir son espace composable. 

et producteurs pour un répertoire en musique pop, à une époque donnée. Prenons par exemple la pop anglaise du tout début des années 1980, avec le cas du groupe Joy Division $^{11}$. Le cas de Joy Division est bien connu, le mythe du groupe s'est construit au gré des livres, films documentaires et fictions biopics qui, jusqu'à nos jours, au fil de la longue traîne de la création de richesse de l'industrie musicale, ont perpétué et transformé le souvenir tragique du chanteur Ian Curtis et la présence dans les bacs des deux albums du groupe, Unknown Pleasures et Closer, ainsi que quelques rares enregistrements live. Ces albums se distinguent par leur singularité emblématique sur le plan sonore et musical ; ils ont fait en leur temps office de référence stylistique de la New Wave anglaise, au moins dans les milieux professionnels de la production.

C'est non pas se laisser aller mais se laisser agir'. C'est selon nous interagir avec cet espace pour faire œuvre, pour produire de la musique, cet espace composé que l'on va donner à entendre.

[...] l'instrumentarium aussi bien théorique que pratique, traditionnel ou récemment développé, donc les instruments de musique avec leur construction caractéristique et les techniques d'exécution qui en découlent, y compris la notation courante ; au-delà également, tous les moyens techniques, les outils, les appareils conceptuels, les techniques de travail développées et exploitées au sein de notre conception et de notre pratique de la musique, de même que les institutions et les marchés concernés au sein de la société10.

Ici l'appareil esthétique rejoint pour ainsi dire la notion de système. qu'objet (la caméra du cinéma, le module de délai du studio), que la façon dont le musicien construit son rapport aux appareils à travers l'espace composable, ou encore dans la globalité de l'appareil esthétique, en tant que réseau, que contexte conceptuel, on est très près d'une approche systémique, qui nous permettrait certainement de renouveler nos méthodologies d'analyse de l'activité musicale. musique électroacoustique, on pourrait s'intéresser à l'usage des appareils dans des projets de production et à ce qui se joue autour.

\section{Pour une approche systémique de la création en industrie musicale}

Une façon de comprendre la singularité de Joy Division consiste à investiguer les rapports entre les jeunes artistes, arrivant en studio avec leurs chansons, et leur producteur, Martin Hannett, qui les accueille et qui a la main sur les appareils d'enregistrement de studio, mais aussi de traitement, en particulier les modules de délai/réverbération ${ }^{12}$, et qui signera la singularité du son de Joy Division ${ }^{13}$ au sein de la légendaire Factory ${ }^{14}$. On pourrait ainsi examiner les rapports de collaboration, de négociation, de pouvoir, d'échange esthétique, de feedback, entre le couple artisteproducteur. L'histoire de la pop donne des exemples nombreux de ce type de couplage, autour des appareils du studio : les Beatles et George Martin assisté de Geoff Emerick, Talking Heads et Brian Eno, Radiohead et Nigel Godrich, etc. Tous ces exemples nous apprennent que la singularité de ces musiques de la reproductibilité se construit dans 
la transformation due au travail des divers agents interagissant, au fil des interactions entre humains, autour d'un appareillage. On pourrait ainsi imaginer une cartographie systémique de ce qui constitue les divers nœuds et relations du système, de la production à la diffusion, en se situant éventuellement en tant que musicien, et en cherchant, éventuellement un changement, notamment dans la relation au public, pour le moment ultra-médiatisé.

L'époque de Joy Division, de Martin Hannett et de la Factory s'éloigne de nous, la crise de l'industrie de la musique est permanente, et le développement de l'autonomie du musicien avec l'accès au Home Studio, puis l'accès à la connaissance experte liée à la numérisation massive, va dans le sens de l'appropriation des appareils, et du développement d'espaces composables propres.

De nos jours, les arts numériques et leur potentiel de connexion et d'interaction nous apportent de nouvelles manières d'écouter, liées au jeu et à l'action.

Dans le cadre du colloque, j'ai ainsi montré mon propre usage détourné d'une Wii Balance (interface Nintendo dédiée au jeu vidéo), connectée au logiciel de synthèse stochastique Cosmos $f^{15}$, pour une musique irreproductible qui se construit en fonction des variations de poids de l'utilisateur au fil de ses mouvements et de sa propre écoute de l'environnement sonore produit par son jeu sur la balance. À l'heure des tablettes numériques, l'audio game se développe à grande vitesse, l'appareil et ses manettes sont au centre du jeu ; mais nous sortons ici du monde du studio électroacoustique, qui était l'objet de l'article...

34 En musique, nous opérons, nous faisons œuvre, dans des contextes culturels divers, selon des styles d'écoute, qui sont culturellement partagés, et qui évoluent avec les mutations technologiques et culturelles. Écouter ou composer, dans les deux cas, c'est faire musique. Écouter est toujours une forme d'action.

Ce que l'on appelle musique se constitue au fil des interactions entre le musicien et son appareillage, dans l'action de l'écoute et du faire. C'est dans cette interaction que se construisent les espaces composables, les espaces d'écoute, les communautés d'écoute, les styles d'écoute, et que s'opèrent les mutations.

En deçà de commentaires esthétiques sur l'époque, et la manière dont celle-ci « apparaît » en étant " appareillée », les appareils, ou appareillages tels qu'observés par Benjamin, correspondent aux mutations technologiques qui, en interaction avec nos appropriations, transforment nos manières de faire, redimensionnent nos espaces composables et modifient notre écoute dans des contextes culturels donnés.

Une réflexion sur l'appareil ne sert alors plus seulement à aborder comment une époque apparaît esthétiquement, mais permet aussi d'examiner la poïétique, les manières de faire et l'ensemble du contexte de production de la musique. Pour une musicologie renouvelée, il s'agirait non plus de faire l'examen de l'œuvre en tant qu'œuvre, mais en tant que pratique, en tant qu'objet/espace musical, produit d'un espace composable, construit en interaction avec l'appropriation des appareils, à travers leur usage créatif, propre à des communautés de pensée et d'écoute dans des contextes culturels donnés, et intégrant éventuellement une perspective systémique. C'est dans cette direction que la pensée de Benjamin peut éclairer encore nos approches. 


\section{BIBLIOGRAPHIE}

Adorno Theodor W., Du fétichisme en musique et de la régression de l'audition, 1938.

Benjamin Walter, «L'œuvre d'art à l'époque de sa reproductibilité technique » (dernière version de 1939), in Euvres III, Maurice de Gandillac (trad.), revue par Rainer Rochlitz, Paris, Gallimard, 2000 , p. 269-316.

Bokesoy Sinan, Macro-Son : situation, évaluation et synthèse du macro-son à l'aide de structures formelles algorithmiques, thèse de doctorat, université Paris 8, 2012.

Carvalho Guilherme, «Formaliser la forme », in Manières de faire des sons, Antonia Soulez (dir.), Horacio Vaggione (dir.), Paris, l'Harmattan, 2010, p. 101-118.

Ingrassia Fanny, Joy Division, la construction d'une identité sonore singulière, une approche méthodologique systémique, mémoire de master 1, université Paris 8, 2013 (recherche en cours).

Lachenmann Helmut, « De la composition » [2005], in Écrits et entretiens, choisis et préfacés par Martin Kaltenecker, Genève, Contrechamps éditions, 2009, p. 129-141.

Lachenmann Helmut, « La question du beau aujourd'hui » [2005], in Écrits et entretiens, Genève, Contrechamps éditions, 2009, p. 69-70.

Nice James, La Factory, grandeur et décadence de Factory Records, Paris, Naïve, 2011.

Robb John, Manchester music city 1976-1996, Paris, Rivages (Rivages Rouges), 2009.

Schaeffer Pierre, Vingt leçons et travaux pratiques destinés aux musiciens mélangeurs, Paris, Radio Française (publication interne), 1938.

Vaggione Horacio, « L'espace composable. Sur quelques catégories opératoires dans la musique électroacoustique », in Jean-Marc Chouvel, Makis Solomos, L'espace : musique/philosophie, Paris, l'Harmattan, 1998, p. 153-166.

Vaggione Horacio, « Perspectives de l'électroacoustique », revue [en ligne] Chimère, $\mathrm{n}^{\circ}$ 40, 2000.

Villa de Almeida André Luis, Réflexions interdisciplinaires sur la perception et la cognition musicales, thèse de doctorat, université Paris 8, 2013.

\section{NOTES}

1. André Luis Villa de Almeida, Réflexions interdisciplinaires sur la perception et la cognition musicales, thèse de doctorat, université Paris 8, 2013, p. 297-300.

2. Walter Benjamin, «L'œuvre d'art à l'époque de sa reproductibilité technique » (dernière version de 1939), in Euvres III, Maurice de Gandillac (trad.), revue par Rainer Rochlitz, Paris, Gallimard, 2000, p. 269-316.

3. Theodor W. Adorno, Du fétichisme en musique et de la régression de l'audition, 1938.

4. Pierre Schaeffer, Vingt leçons et travaux pratiques destinés aux musiciens mélangeurs, Paris, Radio Française (publication interne), 1938.

5. André Luis Villa de Almeida, Réflexions interdisciplinaires sur la perception et la cognition musicales, chap. 9 « Du phonographe aux instruments électroniques », p. 284-322.

6. Identités de la guitare électrique. Communications issues des journées d'étude interdisciplinaires qui se sont tenues les 18 et 19 mai 2009 à la Maison des sciences de l'Homme 
Paris Nord, sous la responsabilité d'Anne Sèdes, Otso Lähdeoja, Benoît Navarret, Santiago Quintans. http://revues.mshparisnord.org/appareil/index.php?id=1009

7. Horacio Vaggione, «L'espace composable. Sur quelques catégories opératoires dans la musique électroacoustique », in Jean-Marc Chouvel, Makis Solomos, L'espace: musique/philosophie, Paris, L'Harmattan, 1998, p. 153-166. Horacio Vaggione, « Perspectives de l'électroacoustique », revue [en ligne] Chimère, $\mathrm{n}^{\circ}$ 40, 2000.

8. Guilherme Carvalho, «Formaliser la forme », in Manières de faire des sons, Antonia Soulez (dir.), Horacio Vaggione (dir.), Paris, L'Harmattan, 2010, p. 101-118.

9. Helmut Lachenmann, « De la composition » [2005], in Écrits et entretiens, choisis et préfacés par Martin Kaltenecker, Genève, Contrechamps éditions, 2009, p. 129-141.

10. Helmut Lachenmann, «La question du beau aujourd'hui » [2005], in Écrits et entretiens, Genève, Contrechamps éditions, 2009, p. 69-70.

11. Je m'appuie ici sur la recherche en cours de Fanny Ingrassia sur « Joy Division, la construction d'une identité sonore singulière, une approche méthodologique systémique ", mémoire de master 1, université Paris 8, 2013.

12. Modules AMS DMX 15-80 Système RMX, Melos EM-110 Echo Chamber, le travail de Martin Hannett est bien connu, ses enregistrements de test et d'expérimentation en studio ayant été publiés sur disque (Joy Division - in the studio with Martin Hannett).

13. John Robb, Manchester music city 1976-1996, Paris, Rivages (Rivages Rouges), 2009 : « Hannett était l'architecte de Joy Division. Impossible de sous-estimer le rôle qu'il a joué sur l'enregistrement du disque. Il a décomposé et rebâti leur musique. Il leur a donné une cohérence et un son qu'ils ont dû apprendre à reproduire sur scène. Ça n'a pas plu au groupe au début, mais il s'est rendu compte par la suite qu'il se passait quelque chose de bien » (Richard Boon, p. 227). «Quand j'ai écouté l'enregistrement d'un concert de Joy Division six mois plus tard, ça n'avait plus rien à voir. Les chansons avaient changées. C'était incroyable. Je ne sais pas ce qui s'est passé, même si je faisais partie du groupe » (Peter Hook, p. 218).

14. James Nice, La Factory, grandeur et décadence de Factory Records, Paris, Naïve, 2011.

15. Logiciel développé par Sinan Bokesoy dans le cadre de sa thèse: Macro-Son: situation, évaluation et synthèse du macro-son à l'aide de structures formelles algorithmiques, université Paris 8 , 2012.

\section{RÉSUMÉS}

On montre en quoi la notion d'appareil empruntée à W. Benjamin peut être utile en musicologie pour développer des approches nouvelles examinant l'activité poïétique des musiciens, en situation de studio, que ce soit en musique expérimentale ou en industrie musicale. On évoque Pierre Schaeffer et la musique concrète, la guitare électrique en tant qu'appareillage, le recours aux espaces composables, ainsi que les perspectives d'une approche systémique notamment pour ce qui concerne l'industrie musicale, avec le cas de Joy Division.

\section{INDEX}

Mots-clés : appareil, musicologie, musique concrète, espace composable, studio électroacoustique, systémique, Joy Division, industrie musicale 
AUTEUR

ANNE SĖDES

Professeur en composition et recherche, Centre de recherche informatique et création musicale (CICM), EA 1572, université Paris 8. 